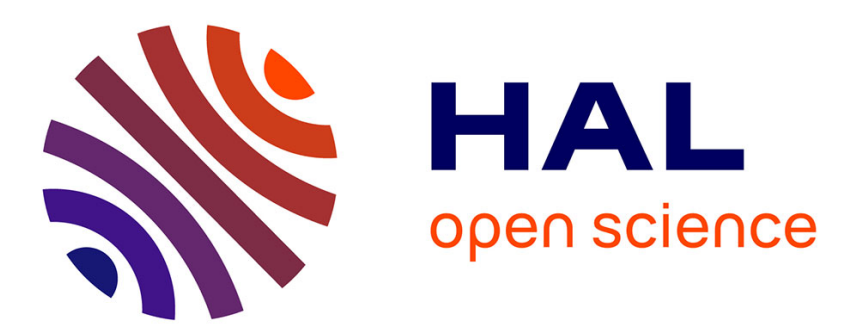

\title{
How to design a recto-verso print displaying different images in various everyday-life lighting conditions
}

\author{
Nicolas Dalloz, Serge Mazauric, Thierry Fournel, Mathieu Hébert
}

\section{To cite this version:}

Nicolas Dalloz, Serge Mazauric, Thierry Fournel, Mathieu Hébert. How to design a recto-verso print displaying different images in various everyday-life lighting conditions. Electronic Imaging Symposium, Jan 2017, Burlingame, CA, United States. pp.33 - 41, 10.2352/ISSN.2470-1173.2017.8.MAAP-289 . hal-01458756

\section{HAL Id: hal-01458756 https://hal.science/hal-01458756}

Submitted on 6 Feb 2017

HAL is a multi-disciplinary open access archive for the deposit and dissemination of scientific research documents, whether they are published or not. The documents may come from teaching and research institutions in France or abroad, or from public or private research centers.
L'archive ouverte pluridisciplinaire HAL, est destinée au dépôt et à la diffusion de documents scientifiques de niveau recherche, publiés ou non, émanant des établissements d'enseignement et de recherche français ou étrangers, des laboratoires publics ou privés. 


\title{
How to design a recto-verso print displaying different images in various everyday-life lighting conditions
}

\author{
Nicolas Dalloz, ${ }^{1}$ Serge Mazauric, ${ }^{2}$ Thierry Fournel, ${ }^{2}$ Mathieu Hébert ${ }^{2}$ \\ ${ }^{1}$ Institut d'Optique - Graduate School, 2 avenue Augustin Fresnel, 91127 Palaiseau, France. \\ ${ }^{2}$ Univ Lyon, UJM-Saint-Etienne, CNRS, Institut d'Optique Graduate School, Laboratoire Hubert Curien UMR 5516, F-42023, Saint- \\ Etienne, France.
}

\begin{abstract}
This study aims at explaining how to design multi-view prints that can show different images in different illumination conditions. A recent reflectance-transmittance optical model for recto-verso halftone prints is extended in order to fasten the calibration step and is used, according to an inversed approach, to design the rectoverso prints displaying the different images in their respective illumination modes. The good prediction accuracy of the model allows creating special effect prints, such as transformation of a binary image in one mode into a second binary image in the second mode, or the transformation of color image into a grey-level version, or the revelation of a message by texture contrast. Regarding the illumination conditions, simultaneous illumination of both sides of the print is allowed. The difficulty of designing such print comes from the fact that the images that are printed on the two sides of the paper are not the images that are displayed, and that the colors displayable in one mode depend on the colors wanted in the other mode. Since no general color management method for these kinds of prints is available, ad hoc methods are proposed for the different effects considered.
\end{abstract}

\section{Introduction}

Books, magazines and most documents are made of paper sheets printed in recto-verso mode. Each page is expected to be viewed in reflection mode; the paper opacity is maximized in order to prevent that various pages are seen simultaneously, which would alter the readability of the content of each page. In contrast, in the present study, we do want to see simultaneously different pages in order to display in transmittance mode content that is not visible on the individual pages in reflection mode. Original visual effects can thus be produced, whose potential for graphical arts has not been exploited yet. The probable reason for this is that no automated color management method is available today for these types of "multiview prints", and their design is not intuitive: the images displayed are different from the digital layouts that are effectively printed. The graphical designer must therefore predict how the light fluxes meeting the recto and verso halftone colors are physically combined, an impossible task without prediction tools.

The multiview imaging concept is well known through the example of lenticular images, varying according to the angle of observation [1]. Other techniques based on special inks or supports can reveal hidden patterns or display different images by varying the illumination or observation conditions: they use the specularity of metallic inks (colors are different in the specular and off-specular directions [2-4]), the anisotropy of line-halftones color on metal supports (colors vary according to the azimuth angle of observation [5]), or fluorescent inks (colors depend on the UV power of the light source [6]). In contrast with these techniques, the one that we present here does not need any special material, source or tool: it can be obtained with standard office paper and general use digital printer.
The spectral reflectance and transmittance model for rectoverso halftone prints necessary to compute the multiview images is presented in Section 2. It is an improved version of the DPRT model presented in Ref. [7], applicable to symmetrical printing supports, which have same optical properties on their two sides. The number of color patches needed for the model's calibration is considerably reduced in this case. Once the model is calibrated, it is possible to compute the amounts of inks to print on the recto and verso sides in order to display the targeted colors in the considered illumination modes.

The first prototypes of multiview recto-verso prints, computed with the original DPRT model, were presented in Ref. [8]. Different patterns were displayed in reflection and transmission modes. In this paper, we extend the principles of multiview images to illumination conditions allowing simultaneous illumination of the two sides, therefore combining the reflection and transmission modes.

The difficulty of designing multiview images by recto-verso printing comes from the limited number of pairs of colors that we can display in the two illumination modes selected. For example, if yellow ink is printed on the recto side, to display a yellow color in the reflectance mode, it is impossible to display a saturated blue color in transmission mode. Likewise, if the color on the recto side is dark, the color in transmission mode is necessarily dark too. The number of displayable pairs of colors is even more limited with the illumination conditions that we address in this paper, where both reflected and transmitted fluxes add. These issues are presented in Section 3, as well as the crucial questions of color matching and registration between the recto and verso layouts.

In Section 4, we present some concepts of visual effects that can be produced: binary multiview images, color-to-grey images and texture contrast images. Section 5 draws the conclusions.

\section{Spectral reflectance-transmittance prediction model for recto-verso prints}

A spectral reflectance and transmittance prediction model, called DPRT model [7], has been recently proposed for printing supports, possibly non-symmetric (i.e., having different optical properties on the recto and the verso sides), printed with halftone colors. This model relies on a discrete 2-flux model for layered materials [9], based on flux transfer matrices. It is somehow an extension of the Clapper-Yule reflection model for multi-ink halftone colors [10], even though the Neugebauer primaries are not characterized by an intrinsic spectral transmittance but considered together with the paper as forming an "inked paper" characterized by a flux transfer matrix. The DPRT model can predict simultaneously, for any pair of halftone colors printed on the recto and verso sides, the transfer factors indicated on Figure 1, namely spectral reflectances $R(\lambda)$ and $R^{\prime}(\lambda)$, and spectral transmittances $T(\lambda)$ and $T^{\prime}(\lambda)$, on the recto and verso sides respectively. 


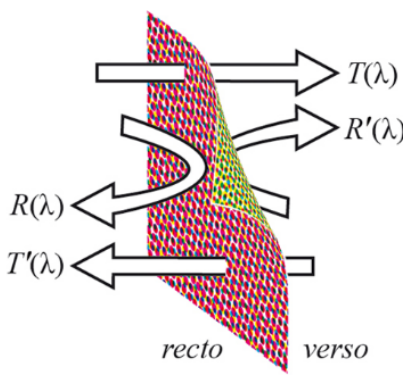

Figure 1. Transfer factors of a recto-verso print.

After several tests carried out with inkjet printer on supercalendered paper and simple office paper, the DPRT model showed its capacity to provide fairly accurate color predictions for rectoverso halftone prints in both reflection and transmission modes [7]. However, the number of color patches to print and the number of measurements to carry out for its calibration is high in comparison to the 44 reflectance measurements needed to calibrate the classical Clapper-Yule model for CMY halftone colors when the dot gain is calibrated according to the method proposed by Hersch and Crété [11,12]: in the DPRT model for CMY halftones, the spectral properties of the inked paper are computed from 64 recto-verso color patches to be measured in reflectance and transmission modes on both recto and verso sides (256 measurements), and the curves assessing dot gain in the halftones, still computed according to the method by Hersch and Crété, need 36 recto-only color patches to be measured in reflectance and transmission modes (72 measurements). This total of 328 measurements, mandatory when the printing support is non-symmetric, can be considerably reduced if the support is symmetric. This model with faster calibration has been called "Double-Layer Reflectance and Transmittance" (DLRT) model. We propose to present it below, after a short recap about the flux transfer matrices that we use.

\section{Flux transfer matrices}

Flux transfer matrices have been recently introduced in the color reproduction domain in order to ease the description of lightmatter interactions in the case of planar layered materials [13]. They relate the incoming and outgoing fluxes at each of the different layers and interfaces within the material. Each component in the material, i.e., layer and interface (labeled $k$ ), is represented by a transfer matrix of the form:

$$
\mathbf{M}_{k}=\frac{1}{T_{k}}\left(\begin{array}{cc}
1 & -R_{k}^{\prime} \\
R_{k} & T_{k} T_{k}^{\prime}-R_{k} R_{k}^{\prime}
\end{array}\right),
$$

where $R_{k}, R_{k}^{\prime}, T_{k}, T_{k}^{\prime}$ represents the four transfer factors of the component, defined in a similar manner as in Figure 1. With spectral transfer factors, a transfer matrix is defined for each waveband.

Consecutive components can also be represented together by one transfer matrix, obtained as the product of the transfer matrices representing the individual components. The matrix multiplication order reproduces the stacking order of the components. For example, the transfer matrix $\mathbf{P}$ representing the stack of three diffusing layers featured in Figure 2, respectively represented by the transfer matrices $\mathbf{M}_{1}, \mathbf{M}_{2}$, and $\mathbf{M}_{3}$, is given by:

$$
\mathbf{P}=\mathbf{M}_{1} \cdot \mathbf{M}_{2} \cdot \mathbf{M}_{3} .
$$

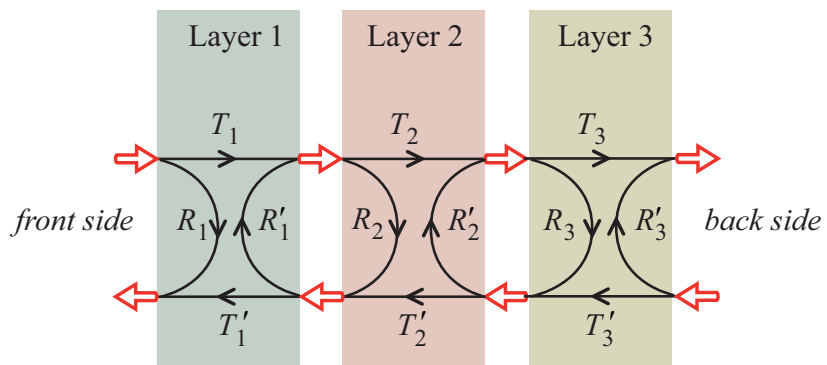

Figure 2. Flux transfers within a stack of three diffusing layers.

Then, we can determine from the entries of matrix $\mathbf{P}=\left\{p_{i j}\right\}$ the four transfer factors of the stack of layers:

$$
\begin{array}{ll}
R=p_{21} / p_{11} & R^{\prime}=-p_{12} / p_{11} \\
T=1 / p_{11} & T^{\prime}=\operatorname{det}(\mathbf{P}) / p_{11}
\end{array}
$$

\section{Printing support}

The printing support is a sheet of strongly scattering medium, e.g., paper, having same optical properties on both sides. It is assumed to have an effective refractive index of 1.5 , with flat interfaces with air. It has been shown in Ref. [14] that rough interfaces do not significantly modify the optical effects of the airpaper interfaces in this case. The paper is modeled by the three component shown in Figure 3: a diffusing paper layer bordered by two interfaces.

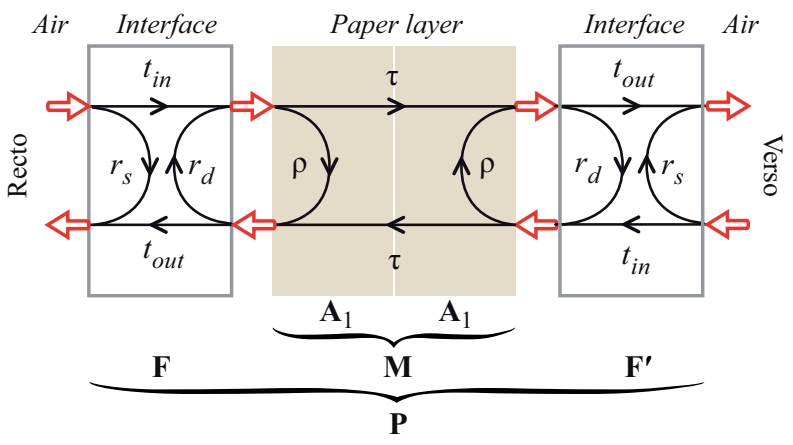

Figure 3. Flux transfers within a paper sheet.

The diffusing paper layer, considered without its interfaces with air, have a reflectance $\rho(\lambda)$ and a transmittance $\tau(\lambda)$. It is represented by a transfer matrix $\mathbf{M}$ defined for each wavelength as:

$$
\mathbf{M}=\frac{1}{\tau}\left(\begin{array}{cc}
1 & -\rho \\
\rho & \tau^{2}-\rho^{2}
\end{array}\right)
$$

The reflectances and transmittances of the interfaces depend on the measuring geometry. In the present study, measurements were done with the X-rite Color i7 spectrophotometer relying on the di: $8^{\circ}$ geometry in reflection mode (illumination with perfectly diffuse light over the hemisphere, and observation at $8^{\circ}$ from the normal of the sample by including the specular reflection component), and the $\mathrm{d}: 0^{\circ}$ geometry in transmission mode (illumination with perfectly 
diffuse light over the hemisphere, and observation in the normal of the sample). The corresponding transfer factors are given in Figure 2. For these geometries and the refractive index value 1.5 , the transfer matrix representing the interface at the recto side is:

$$
\mathbf{F}=\frac{1}{t_{\text {in }}}\left(\begin{array}{cc}
1 & -r_{d} \\
r_{s} & t_{\text {in }} t_{\text {out }}-r_{s} r_{d}
\end{array}\right)=\left(\begin{array}{cc}
1.111 & -0.667 \\
0.044 & 0.403
\end{array}\right),
$$

and the one representing the interface at the verso side is:

$$
\mathbf{F}^{\prime}=\frac{1}{t_{\text {out }}}\left(\begin{array}{cc}
1 & -r_{s} \\
r_{d} & t_{\text {in }} t_{\text {out }}-r_{s} r_{d}
\end{array}\right)=\left(\begin{array}{cc}
1.111 & -0.044 \\
0.667 & 0.403
\end{array}\right) \text {. }
$$

Once the spectral reflectance $R(\lambda)$ and transmittance $T(\lambda)$ of the paper sheet are measured, we can compute a transfer matrix $\mathbf{P}$ representing the paper sheet with its interfaces:

$$
\mathbf{P}=\frac{1}{T}\left(\begin{array}{cc}
1 & -R \\
R & T^{2}-R^{2}
\end{array}\right)
$$

then a transfer matrix $\mathbf{M}$ representing the paper layer without the interfaces, thanks to the following matrix equation:

$$
\mathbf{M}=\mathbf{F}^{-1} \cdot \mathbf{P} \cdot \mathbf{F}^{-1} \text {. }
$$

The reduction of needed calibration measurements comes from the idea of subdividing the paper layer into two identical sublayers, both represented by the transfer matrix:

$$
\mathbf{A}_{1}=\mathbf{M}^{1 / 2}
$$

\section{Solid colors}

In CMY printing, one has eight Neugebauer primaries: white (no ink), cyan, magenta, yellow, red (magenta and yellow), green (cyan and yellow), blue (cyan and magenta) and black (cyan, magenta and yellow).

Let us print the recto-side of the paper with a solid color $(100 \%$ surface coverage) corresponding to one of seven Neugebauer primaries containing at least one ink, labeled $i=2, \ldots, 8 \quad(i=1$ denotes the "white" primary, i.e., the unprinted paper). We measure the four transfer factors $R_{i}(\lambda), T_{i}(\lambda), R_{i}^{\prime}(\lambda)$, and $T_{i}^{\prime}(\lambda)$. The matrix $\mathbf{P}_{i 1}$ representing this primary (printed on the recto side only) is given for each wavelength by:

$$
\mathbf{P}_{i 1}=\frac{1}{T_{i}}\left(\begin{array}{cc}
1 & -R_{i}^{\prime} \\
R_{i} & T_{i} T_{i}^{\prime}-R_{i} R_{i}^{\prime}
\end{array}\right) .
$$

We can deduce the matrix $\mathbf{M}_{i 1}$ representing the inked paper without interfaces:

$$
\mathbf{M}_{i 1}=\mathbf{F}^{-1} \cdot \mathbf{P}_{i 1} \cdot \mathbf{F}^{-1}
$$

where matrices $\mathbf{F}$ and $\mathbf{F}^{\prime}$ have same meaning as in Eq. (8).

As for the unprinted paper, we subdivide the inked paper into two sublayers (see Figure 4), one for the recto side containing the inks, represented by a matrix $\mathbf{A}_{i}$, and one for the verso side, similar to the sublayers present in the unprinted paper, therefore represented by the matrix $\mathbf{A}_{1}$ given by Eq. (9). We thus have:

$$
\begin{aligned}
\mathbf{A}_{i} & =\mathbf{M}_{i 1} \cdot \mathbf{A}_{1}^{-1} \\
& =\mathbf{F}^{-1} \cdot \mathbf{P}_{i 1} \cdot \mathbf{F}^{\prime-1} \cdot \mathbf{M}^{-1 / 2}
\end{aligned}
$$

Matrix $\mathbf{A}_{i}$ is to be computed for each wavelength, and for each of the seven Neugebauer primaries containing at least one ink. The matrix $\mathbf{A}_{i}$ for the Neugebauer primary "white" (no ink) is simply the matrix $\mathbf{A}_{1}$ given by Eq. (9). The transfer factors of the inked sublayer are computed from the entries of $\mathbf{A}_{i}=\left\{p_{i j}\right\}$ by similar equations as Eq. (3):

$$
\begin{array}{ll}
\rho_{i}=p_{21} / p_{11}, & \rho_{i}^{\prime}=-p_{12} / p_{11}, \\
\tau_{i}=1 / p_{11}, & \tau_{i}^{\prime}=\operatorname{det}\left(\mathbf{A}_{i}\right) / p_{11} .
\end{array}
$$

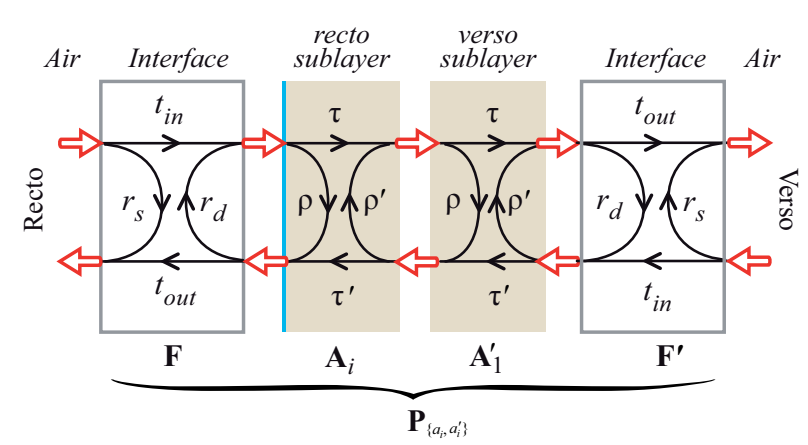

Figure 4. Flux transfers within a paper sheet printed with a solid primary on the recto side.

\section{Recto-verso halftone colors}

For a halftone color printed on the recto side of the paper, with effective surface coverages $a_{i}$ for the eight Neugebauer primaries $i$, we also consider the inked paper without interfaces, and more precisely the sublayer on the recto side which contains the inks (the other sublayer being unprinted paper, therefore represented by the matrix $\mathbf{A}_{1}$ introduced previously). The four transfer factors of this recto-side sublayer are functions of their equivalent $\rho_{i}, \rho_{i}^{\prime}, \tau_{i}$, and $\tau_{i}^{\prime}$ for the eight Neugabeur primaries considered separately, given by Eq. (13):

$$
\begin{array}{ll}
\rho_{\left\{a_{i}\right\}}=\sum_{i=1}^{8} a_{i} \rho_{i} & \rho_{\left\{a_{i}\right\}}^{\prime}=\sum_{i=1}^{8} a_{i} \rho_{i}^{\prime} \\
\tau_{\left\{a_{i}\right\}}=\sum_{i=1}^{8} a_{i} \tau_{i} & \tau_{\left\{a_{i}\right\}}^{\prime}=\sum_{i=1}^{8} a_{i} \tau_{i}^{\prime}
\end{array}
$$

The recto-side sublayer is represented by the transfer matrix:

$$
\mathbf{A}_{\left\{a_{i}\right\}}=\frac{1}{\tau_{\left\{a_{i}\right\}}}\left(\begin{array}{cc}
1 & -\rho_{\left\{a_{i}\right\}}^{\prime} \\
\rho_{\left\{a_{i}\right\}} & \tau_{\left\{a_{i}\right\}} \tau_{\left\{a_{i}\right\}}^{\prime}-\rho_{\left\{a_{i}\right\}} \rho_{\left\{a_{i}\right\}}^{\prime}
\end{array}\right) .
$$

If a halftone color is printed on the verso side, with effective surface coverages $a_{i}^{\prime}$ for the eight Neugebauer primaries $i$, the verso-side sublayer has the following transfer factors, again functions of the ones given by Eq. (13) thanks to the symmetry of the printing setup: 


$$
\begin{array}{ll}
\rho_{\left\{a_{i}^{\prime}\right\}}=\sum_{i=1}^{8} a_{i}^{\prime} \rho_{i} & \rho_{\left\{a_{i}^{\prime}\right\}}^{\prime}=\sum_{i=1}^{8} a_{i}^{\prime} \rho_{i}^{\prime} \\
\tau_{\left\{a_{i}^{\prime}\right\}}=\sum_{i=1}^{8} a_{i}^{\prime} \tau_{i} & \tau_{\left\{a_{i}^{\prime}\right\}}^{\prime}=\sum_{i=1}^{8} a_{i}^{\prime} \tau_{i}^{\prime}
\end{array}
$$

The verso-side sublayer is represented by the transfer matrix:

$$
\mathbf{A}_{\left\{a_{i}^{\prime}\right\}}^{\prime}=\frac{1}{\tau_{\left\{a_{i}^{\prime}\right\}}^{\prime}}\left(\begin{array}{cc}
1 & -\rho_{\left\{a_{i}^{\prime}\right\}} \\
\rho_{\left\{a_{i}^{\prime}\right\}}^{\prime} & \left.\tau_{\left\{a_{i}^{\prime}\right\}}^{\prime} \tau_{\left\{a_{i}^{\prime}\right\}}-\rho_{\left\{a_{i}^{\prime}\right\}}^{\prime} \rho_{\left\{a_{i}^{\prime}\right\}}\right\}
\end{array}\right) .
$$

Finally, the transfer matrix representing the recto-verso print, whose structure is shown in Figure 5, is obtained by multiplying the transfer matrices of the recto-side interface, the recto-side sublayer, the verso-side sublayer and the verso-side interface:

$$
\mathbf{P}_{\left\{a_{i}, a_{i}^{\prime}\right\}}=\mathbf{F} \cdot \mathbf{A}_{\left\{a_{i}\right\}} \cdot \mathbf{A}_{\left\{a_{i}^{\prime}\right\}}^{\prime} \cdot \mathbf{F}^{\prime}
$$

The global transfer factors of the recto-verso print are deduced from $\mathbf{P}_{\left\{a_{i}, a_{i}^{\prime}\right\}}=\left\{p_{i j}\right\}$ by similar equations as Eq. (3).

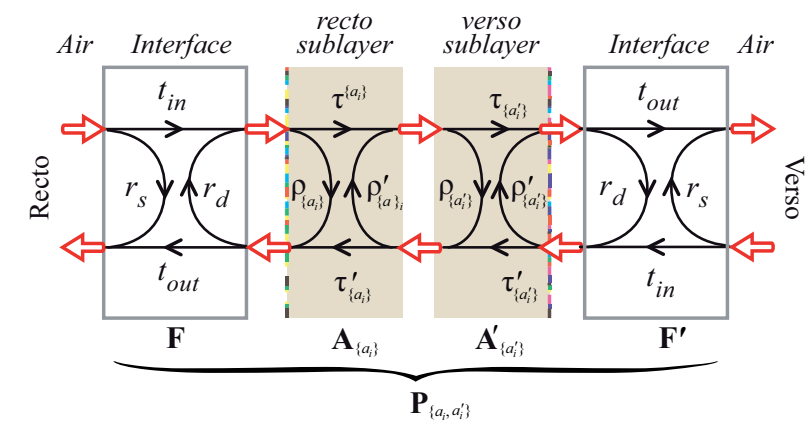

Figure 5. Flux transfers within a recto-verso halftone print.

On each side, the conversion of nominal surface coverages ( $c$, $m, y)$ for the three inks cyan, magenta and yellow, into effective surface coverages for the eight Neugebaeur primaries, relies on twelve nominal-to-effective surface coverage functions according to the method recommended by Hersch and Crété [11], and on the Demichel equations (see details in Ref. [12]).

The calibration of the model needs $44 \mathrm{CMY}$ patches printed on the recto only, the same as for reflectance-only models [12]: 8 solid color patches for the 8 Neugebauer primaries, whose spectral reflectances and transmittances are measured on both the recto and verso sides, and 36 halftone patches required to compute the twelve nominal-to-effective ink surface coverage functions, whose spectral reflectance and transmittance are measured on the recto side. This makes a total of 104 measurements, therefore lower than the 328 measurements needed by the DPRT model [7].

\section{Prediction accuracy}

The model was tested on a few tens of different recto-verso halftone samples printed with the Xerox Phaser 6500 electrophotographic printer and a standard $80 \mathrm{~g} / \mathrm{m}^{2}$ office paper. The prediction accuracy is assessed by the average CIELAB $\Delta \mathrm{E}_{94}$ value computed between the measured and predicted spectra, by converting them first into CIE 1931 XYZ tristimulus values, calculated with a D65 illuminant, and then into CIE $1976 \mathrm{~L}^{*} \mathrm{a}^{*} \mathrm{~b}^{*}$ color coordinates [15], using as white references a perfectly white reflector (unit reflectance) or transmitter (unit transmittance). The average $\Delta \mathrm{E}_{94}$ values obtained in pure reflectance and pure transmission modes are shown in Table 1 . By way of comparison, we also give the average $\Delta \mathrm{E}_{94}$ values obtained for the same set of samples by two other models: the Duplex Clapper-Yule model [7], which is a direct extension of the multi-ink Clapper-Yule model to recto-verso halftone prints, and the Yule-Nielsen model used in reflection mode [16,11] or in transmission mode [17].

The DLRT model is slightly less accurate than the other two models in reflection mode, but is it noticeably more accurate in transmission mode, which is the key point for the visual effects that we show in the next section.

Table 1. Compared prediction accuracy between three models*

\begin{tabular}{lcc} 
& Reflectance & Transmittance \\
\hline \hline DLRT model & 1,39 & 0.89 \\
Duplex Clapper-Yule model & 1.27 & 2.31 \\
Yule-Nielsen model & $1.35(n=2.4)$ & $1.38(n=1.4)$ \\
\hline \hline
\end{tabular}

*Average $\Delta E_{94}$ value computed between predicted and measured spectra

\section{Doubled paper}

Without any additional calibration measurement, the model is able to predict the reflectance and transmittance of recto-verso colors on a doubled paper sheet (e.g. a paper sheet folded in two), by simply replacing Eq. (18) with the following one:

$$
\mathbf{P}_{\left\{a_{i}, a_{i}^{\prime}\right\}}=\mathbf{F} \cdot \mathbf{A}_{\left\{a_{i}\right\}} \cdot \mathbf{A} \cdot \mathbf{F}^{\prime} \cdot \mathbf{F} \cdot \mathbf{A} \cdot \mathbf{A}_{\left\{a_{i}^{\prime}\right\}}^{\prime} \cdot \mathbf{F}^{\prime}
$$

\section{Inverse approach: displaying different images under different illumination conditions}

Once the model is calibrated and its prediction accuracy has been verified, we can use it in an inversed mode to produce multiview prints, i.e., prints displaying different images according to the illumination and observation configurations. For example, we can illuminate the print on the observer's side (reflection mode) or the opposite side (transmission mode).

The first prototypes of multiview recto-verso prints were presented in Ref. [8]. Different patterns were displayed in reflection mode (only the recto appears) and in transmission mode (the recto and verso images are mixed). Pure transmission mode means that only the verso side is illuminated, while the recto side receives no light. This illumination mode is rather constraining because it requires to be in a very dark room: if ambient light slightly illuminates the recto side, the displayed colors are not the targeted ones.

\section{Illumination modes}

In order to allow a certain amount of ambient light in the transmission mode, we define illumination conditions where the print is simultaneously illuminated on both sides, with spectral irradiances having similar spectral distributions but different magnitudes. These irradiances can be written $(1-\alpha) E_{i}(\lambda)$ for the recto side and $\alpha E_{i}(\lambda)$ for the verso side, where $\alpha$ is a number between 0 and 1 . The observer, located in front of the recto side, perceives a spectral radiance defined as follows if we assume that the print is nearly Lambertian: 


$$
L_{\alpha T}(\lambda)=\frac{1}{\pi}\left[(1-\alpha) R(\lambda) E_{i}(\lambda)+\alpha T^{\prime}(\lambda) E_{i}(\lambda)\right]
$$

where $R(\lambda)$ and $T^{\prime}(\lambda)$ denote respectively the recto-side reflectance and the verso-to-recto transmittance of the print.

In the next sections, we will consider two values for $\alpha$ : - $\alpha=0$, which corresponds to the pure reflection mode. The radiance perceived by the observer, depends only on the amount of inks printed on the recto side, is:

$$
L_{R}(\lambda)=\frac{1}{\pi} R(\lambda) E_{i}(\lambda)
$$

- $\alpha=0.9$, which means that the irradiance on the verso is 9 times the irradiance on the recto. This configuration corresponds to the lighting conditions of a window with overcast sky on the one side, and ambient light in a small room without artificial lighting on the other side. This mode is referred to as " $\alpha$-transmission mode".

\section{Multiview recto-verso prints: main issues}

The ink quantities to print on the recto and verso sides are obtained by performing a brute-force optimization in order to obtain the targeted CIE $1976 \mathrm{~L}^{*} \mathrm{a}^{*} \mathrm{~b}^{*}$ values, or the targeted spectral radiance, in the two selected illumination modes. We generally compute first the ink surface coverages on the recto side yielding the reflection-mode image, then the ones on the verso side so as to obtain the targeted image in $\alpha$-transmission mode.

However, the computation of multiview images remains delicate, mainly because the CMY images that are transferred onto paper are not the ones that we want to display. The CMY layouts to print on the recto and the verso sides are interdependent. This is a rather unusual configuration in graphical arts, where the digital layout is generally similar to the physical image that we will see once printed. Moreover, the graphic designer must cope with the issues listed below.

\section{Conditional color gamut}

The ink surface coverages on the recto side determine the set of colors that can be displayed in $\alpha$-transmission mode, which is referred to as conditional gamut. The size of a conditional gamut depends on the halftone color on the recto side, as well as the transmittance of the paper. The standard office paper that we use has a transmittance around $15 \%$, whereas its reflectance is around $80 \%$. With the $\alpha$ value of 0.9 that we considered, the radiances reflected and transmitted by the paper have comparable orders of magnitude; the coloration due to the inks on the recto side therefore tends to predominate on the coloration due to the inks on the verso, thus limiting the gamut displayable in $\alpha$-transmission mode.

Figure 6 shows an example of conditional gamuts for the $\alpha-$ transmission mode corresponding to two colors printed on the recto side. These conditional gamuts have been generated by predicting the spectral radiances perceived in $\alpha$-transmission mode for each of the colors selected on the recto side, represented by certain surface coverages for the cyan magenta and yellow inks, and by considering 1000 halftone colors on the verso side, randomly selected. The spectral radiances are then converted into CIE 1931 XYZ tristimulus values, calculated with a D65 illuminant, and then into CIE 1976 $\mathrm{L}^{*} \mathrm{a} \mathrm{b}^{*}$ color coordinates using as white reference the spectral radiance of the unprinted paper in this illumination mode.
The obtained points in the CIE1976 L*a*b* space are represented on the $\left(a^{*}, b^{*}\right)$ - and $\left(L^{*}, C^{*}\right)$-planes, where $C^{*}$ denotes the chroma coordinate given by $C^{*}=\sqrt{a^{* 2}+b^{* 2}}$.
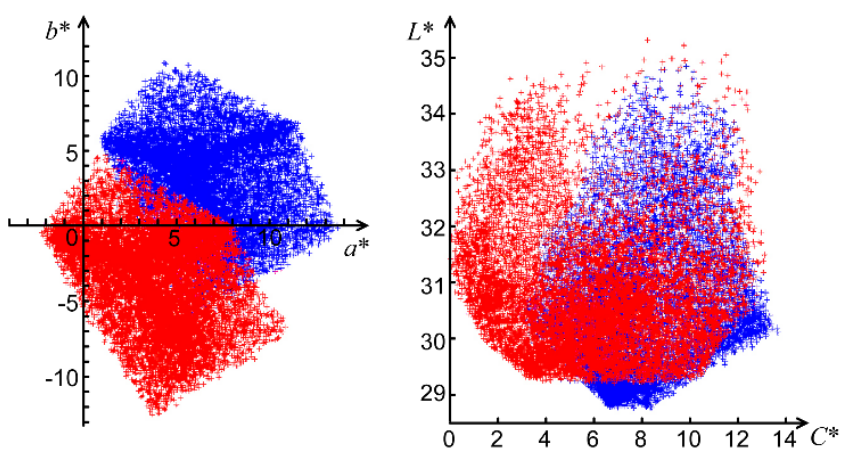

Figure 6. Conditional color gamuts in $\alpha$-transmission mode that would be obtained by printing the halftone colors $(c, m, y)=(0.1,0.1,0.1)$ (blue dots) and $(0.2,0.1,0.1)$ (red dots) on a doubled office paper (electrophotographic printing). The conditional gamuts are represented in the $\left(a^{*}, b^{*}\right)$ - and $\left(L^{*}, C^{*}\right)$ planes of the CIE $1976 L^{*} a^{*} b^{*}$ color space.

\section{Color matching}

It is possible that areas expected to display one color in $\alpha$ transmission mode must display different colors in reflection mode. The ink surface coverages therefore vary over these areas, on both the recto and verso sides. To be sure that uniform color is displayed in $\alpha$-transmission mode, we must ascertain that the spectral radiances $L_{\alpha T}(\lambda)$ issued from all the pixels in these areas are metameric.

In practice, since we use the same three inks on the recto and verso sides, we can reproduce almost spectrally the radiance $L_{\alpha T}(\lambda)$ in all areas where the targeted color is reproducible. We thus have a spectral matching. The rms deviation between the different spectral radiance suffices to assess their similarity. We can also use a color difference metric, for example the CIE $\Delta$ E94.

Moreover, being given the three ink surface coverages on the recto side, the ink surface coverage permitting to display a given reproducible color in $\alpha$-transmission mode have unique values. This is not true with more than three inks: ink selection strategies should be used, as the Under Color Removal (UCR) methods for black ink management in CMYK printing [18].

\section{Registration between the recto and verso images}

The color matching quality does not only depend on the accuracy of the prediction model and the relevance of the color metric used to match the colors of neighboring pixels, but also on the registration between the recto and verso images, which is often a challenging issue in printing. Imperfect registration can create ghost images in the $\alpha$-transmission mode. The human visual system is capable of perceiving lines or contours thinner than $100 \mu \mathrm{m}$, especially when they are well contrasted and surrounded by a uniform background. This length is much smaller than the mechanical accuracy of most duplex printers. This is why in the example presented here, we printed the recto and verso images on different paper sheets and superposed them manually. The printing support, in this case, is a doubled paper sheet. The model, calibrated with recto-only color patches printed on a single sheet, allows predicting the reflectance and transmittance of printed doubled papers sheets as explained at the end of Section 2. The advantage of 
doubling the thickness of the support is that its point spread function in transmission mode is increased, thus limiting the visibility of slightly misregistrated contours. However, the doubled paper being more opaque (its transmittance is less than 10\%), the conditional gamuts in $\alpha$-transmission mode are consequently reduced.

\section{Concepts of multiview images}

Ideal multiview print would display any color image in one illumination mode, and any other image in the other illumination mode. However, due to the limitations explained in the previous section, this kind of print cannot be achieved by recto-verso printing. In this section, we propose some concepts of images that can be produced: the display of different binary images, the display of one image with different colors (e.g. multicolor in one mode, and grey in the other mode), or the revelation in transmission mode of uniformly colored patterns in a textured background.

\section{Binary images}

The easiest design option for multiview prints is based on binary images, thus displaying two colors in each illumination mode. Two colors, denoted as $X_{1}$ and $X_{2}$, are selected to be displayed in reflection mode. They are specified by the ink surface coverages on the recto side, $\mathbf{a}_{u}=\left(c_{u}, m_{u}, y_{u}\right), u \in\{1,2\}$. Two other colors, $Y_{1}$ and $Y_{2}$, are also selected for the $\alpha$-transmission mode. They are reproduced by four different halftones, with ink surface coverages denoted as $\mathbf{a}_{u, v}^{\prime}=\left(c_{u, v}^{\prime}, m_{u, v}^{\prime}, y_{u, v}^{\prime}\right), \quad(u, v) \in\{1,2\}$, corresponding to areas where color $X_{u}$ is expected in reflection mode and color $Y_{v}$ is expected in $\alpha$-transmission mode. These ink surface coverages must satisfy the following conditions:

$$
\begin{array}{ll}
L_{R}\left(\mathbf{a}_{1}\right) \equiv X_{1}, & L_{\alpha T}\left(\mathbf{a}_{1}, \mathbf{a}_{1,1}^{\prime}\right) \equiv L_{\alpha T}\left(\mathbf{a}_{2}, \mathbf{a}_{2,1}^{\prime}\right) \equiv Y_{1}, \\
L_{R}\left(\mathbf{a}_{2}\right) \equiv X_{2}, & L_{\alpha T}\left(\mathbf{a}_{1}, \mathbf{a}_{1,2}^{\prime}\right) \equiv L_{\alpha T}\left(\mathbf{a}_{2}, \mathbf{a}_{2,2}^{\prime}\right) \equiv Y_{2} .
\end{array}
$$

where $L_{R}(\mathbf{a})$ denotes the spectral radiance observed in reflection mode when a halftone with ink surface coverages a are printed on the recto, $L_{\alpha T}\left(\mathbf{a}, \mathbf{a}^{\prime}\right)$ the radiance observed in $\alpha$-transmission mode when halftones with ink surface coverages a and a' are printed on the recto side, respectively the verso side, and symbol " $\equiv X$ " means "yields the perceived color $X$ ".

In order to be sure that the four pairs of colors $\left(X_{u}, Y_{v}\right)$ can be reproduced, we can verify that the colors $Y_{v}$ belong to the conditional gamuts in $\alpha$-transmission mode for the selected ink surface coverages $x_{u}$ on the recto side. We can also just check that the color distance $\Delta \mathrm{E}_{94}$ between the radiances $L_{\alpha T}\left(\mathbf{a}_{1}, \mathbf{a}_{1, u}^{\prime}\right)$ and $L_{\alpha T}\left(\mathbf{a}_{2}, \mathbf{a}_{2, u}^{\prime}\right)$ is small, hopefully below 1 unit.

In practice, the ink surface coverages $\mathbf{a}_{1}, \mathbf{a}_{2}, \mathbf{a}_{1,1}^{\prime}$ and $\mathbf{a}_{1,2}^{\prime}$ can be chosen, and the ink surface coverages $\mathbf{a}_{2,1}^{\prime}$ and $\mathbf{a}_{2,2}^{\prime}$ computed by using the DLRT prediction model in inversed mode in order to satisfy conditions (22) at best.

Notice that the displayed image in $\alpha$-transmission mode may be composed of large uniform areas. The registration between the recto and the verso images is therefore critical.

The print shown in Figure 7 displays a text in reflection mode which disappears by color matching in $\alpha$-transmission mode, while another text, printed in black on the verso side, appears. (a)

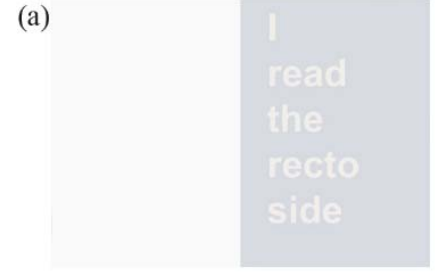

(b)

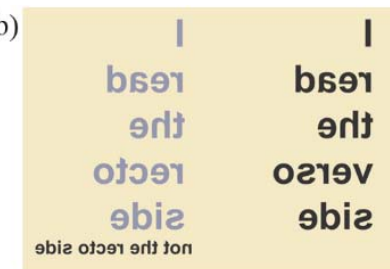

(c)

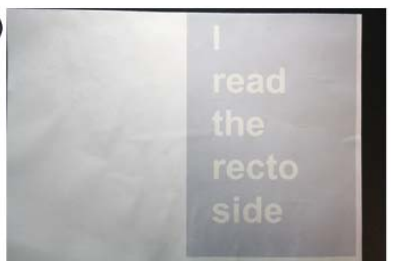

(d)

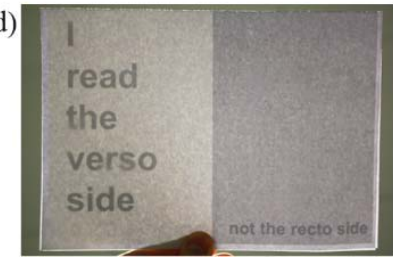

Figure 7. Multiview recto-verso print based on binary images. Top row: digital layouts for (a) the recto side, (b) the verso side. Bottom row: Pictures of the print in (c) reflection mode and (d) in 0.9-transmission mode. (a)

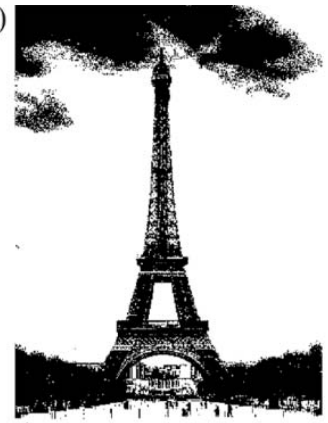

(c)

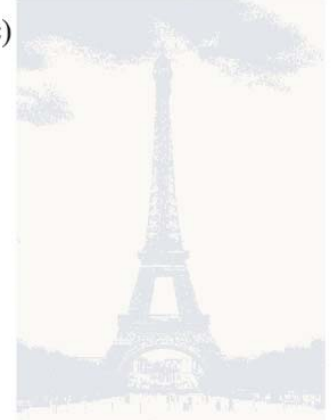

(e)

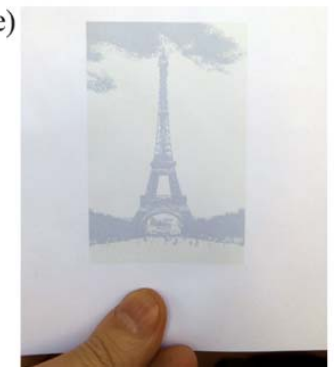

(b)

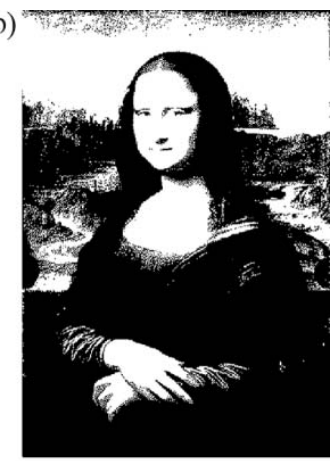

(d)

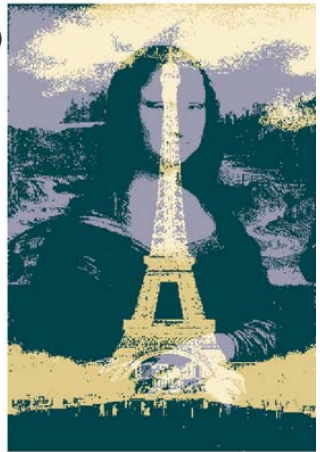

(f)

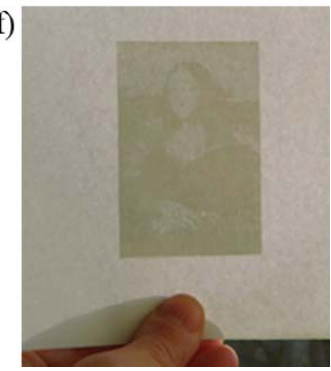

Figure 8. Multiview recto-verso print based on binary images, representing the Eiffel tower in reflection mode, and Mona Lisa in a-transmission mode. Top row: binary images to be displayed (a) in reflection mode, (b) in 0.9transmission mode; middle row: digital layouts for (c) the recto side, (d) the verso side, bottom row: (e) Picture of the print in 0.9-transmission mode. 
(a)
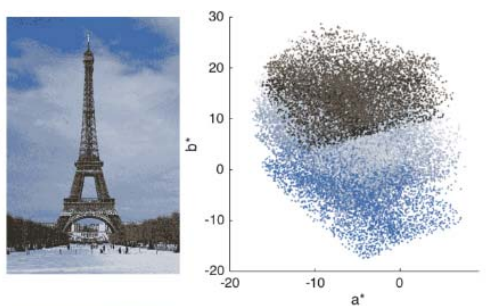

(b)
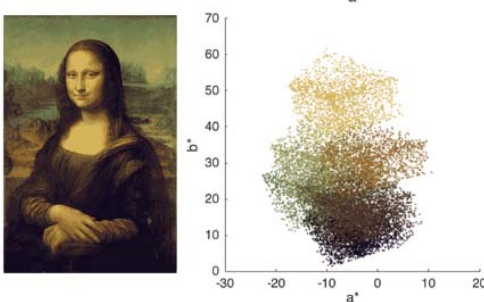
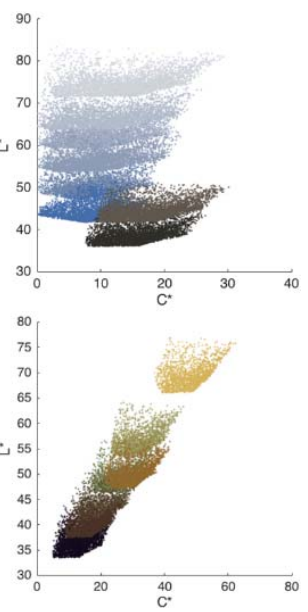

Figure 9. Quantized images of (a) the Eiffel Tower, and (b) Mona Lisa, containing each one eight colors, and corresponding conditional color gamuts in $\alpha$-transmission mode that would be obtained if each of these eight colors was printed on the recto side of a double office paper (electrophotographic printing). The conditional gamuts are represented by 1000 points randomly selected and plotted in the $\left(a^{*}, b^{*}\right)$ - and $\left(L^{*}, C^{*}\right)$-planes of the CIE1976 $L^{*} a^{*} b^{*}$ color space with the corresponding color among the eight colors.

\section{Contrast in the binary images}

The two colors $X_{1}$ and $X_{2}$ selected on the recto side must generate conditional gamuts in $\alpha$-transmission mode that partially meet each other in order to be sure that two colors $Y_{1}$ and $Y_{2}$ can be found in their intersection. If we want a good contrast in reflection mode, colors $X_{1}$ and $X_{2}$ must be far from each other in the color space, but the intersection of their conditional gamuts may be small, or even empty. This is illustrated by Figure 9, which shows a digital layout for the recto side (displayed in reflectance mode) containing eight colors $X_{i}$, as well as the conditional gamuts that would be obtained in $\alpha$-transmission mode if each of these eight colors was printed on a doubled office paper. We see that the conditional gamuts associated with the darkest and brightest colors are disjoint. Therefore, the colors $X_{1}$ and $X_{2}$ should be sufficiently close to each other in order to generate conditional gamuts with non-empty intersection. Moreover, the intersection should be large enough to select well distinct colors $Y_{1}$ and $Y_{2}$ and obtain a sufficient contrast in $\alpha$-transmission mode.

A good contrast therefore implies a poor contrast in $\alpha$ transmission mode (or even the impossibility to display a binary image), and reciprocally a good contrast in $\alpha$-transmission mode means that the two colors on the recto side are close to each other, therefore that the contrast in reflectance mode is poor. There is a trade-off to find in order to have enough contrast in the two modes.

In practice, optimal contrast is reached when the total amounts of inks on the recto and verso sides (i.e., $c+m+y+c^{\prime}+m^{\prime}+y^{\prime}$ ) are as different as possible between the different areas. This total amount of inks has a strong influence on the lightness of the colors, while the human visual systems seems to me more sensible to lightness variations than hue or chroma variations in an image [19].

\section{Color-to-grey images}

A second type of multi-view image, after binary images, consists in displaying a color image in reflection mode and an achromatic version of this image in transmission mode.

In order to simplify the explanations of the principles, we propose to consider a quantized image containing eight colors, like the ones shown in Figure 9. Each of these eight colors, printed on the recto side with their respective ink surface coverages $\mathbf{a}_{i}=\left(c_{i}, m_{i}, y_{i}\right)$, will be turned into an achromatic color on the grey axis, characterized by values $a^{*}=b^{*}=0$ and a given lightness value $L^{*}$ in the CIE $1976 L^{*} a^{*} b^{*}$ space. The lightness of the grey color is expected to be similar to the one of the original colors.

Color-to-grey effect can be achieved only if the eight conditional gamuts, associated with the eight halftone colors printed on the recto side, meet the grey axis. We select in each conditional gamut a reproducible lightness value, as close as possible to the lightness of the original color. The ink surface coverages on the verso side are computed in order to obtain these achromatic colors.

We can see from Figure 9 that the Mona Lisa image is not adapted to the color-to-grey effect: no conditional gamut meets the grey axis (ordinate axis in the right-most graph). The Eiffel Tower image is more adapted, even though the two conditional gamuts associated with the darkest halftones on the recto do not meet the grey axis. In this case, we select the color with lowest chroma.

The grey-to-level print computed from the Eiffel Tower image is shown in Figure 10. We observe, as expected, that the dark areas remain slightly chromatic in $\alpha$-transmission mode. (a)

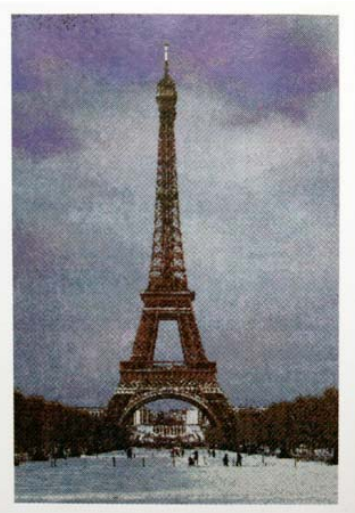

(b)

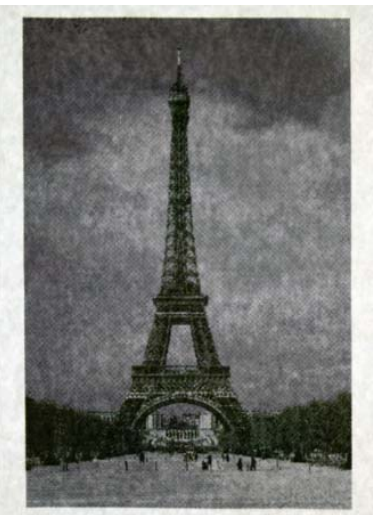

Figure 10: Pictures of a multiview recto-verso print presenting a color-to-grey effect: (a) image displayed in reflection mode, (b) image displayed in 0.9transmission mode.

\section{Texture contrast images}

A third type of multiview image that can be produced with recto-verso prints relies on the perceived contrast between uniform and textured areas in an image, permitting to distinguish patterns. In the following example, shown in Figure 11, the two images printed on the resto and verso sides are a tessellation of eight colors, distributed randomly. The colors on the recto side are arbitrarily chosen, making sure that the corresponding conditional gamuts in $\alpha$-transmission mode have a non-empty intersection. In this intersection, a color is selected, which will fill the patterns to reveal in $\alpha$-transmission mode. The eight halftone colors on the verso side are then computed in order to obtain this $\alpha$-transmission color. In the pattern, each selected color on the recto side faces the computed color on the verso side. Outside the pattern, the colored squares are rearranged in order to obtain a color texture. If the rearrangement is random, since no information is contained in the recto- and versoimages and a message is revealed in $\alpha$-transmission mode, we have a visual cryptography scheme similar to the one based on printed films presented in Ref. [20], extending to color the principles 
introduced by Naor and Shamir with black and white images [21]. We can also use custom rearrangement rules in order to increase the perceived texture outside the message, and thus maximize the texture contrast between the message and the background. We can consider the obtained print as an example of stenographic image.

(a)

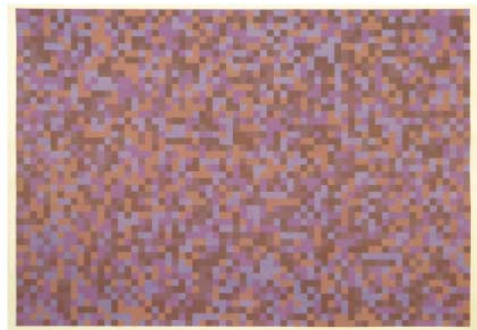

(b)

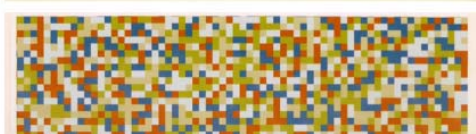

(c)

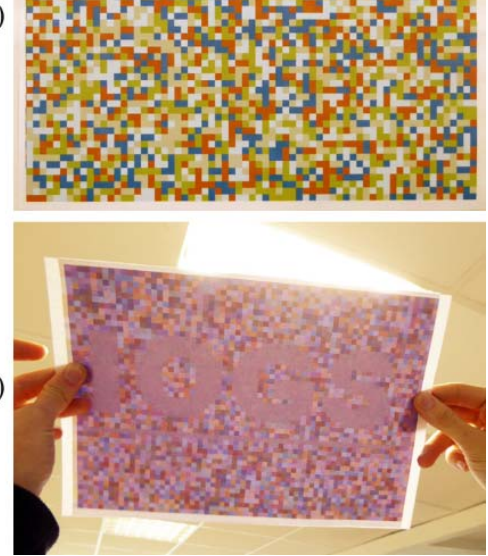

Figure 11: Pictures of a steganographic print based on texture contrast: (a) recto-side image and (b) verso-side image displayed in reflection mode, (c) image displayed in 0.9-transmission mode.

\section{Conclusions}

We introduced in the present paper a model based on flux transfer matrices capable of predicting the spectral reflectance and transmittance of recto-verso halftone prints by means of 104 measurements for its calibration, a rather reasonable number in comparison with previous models. The accuracy of the model is sufficiently good to address the inverse approach, which consists in computing the ink surface coverages in the recto-side and verso-side halftone colors in order to display targeted images under different illumination conditions. We thus obtain multiview prints. The illumination modes considered in our examples are the reflectance mode, where only the recto side is illuminated, and a so-called $\alpha$ transmission mode, where the print receives $10 \%$ of the total irradiance on the recto side, and $90 \%$ on the verso side, the observer being in front of the recto side. The design of multiview images is made difficult by the fact that the number of pairs of colors displayable by one recto-verso color patch in the two modes is rather limited. The main issues are discussed in this paper, and different strategies permitting to design prints with interesting visual effects are proposed: the first one is based on binary color images, the second one transforms a color image into an achromatic version of this image, and the third one is based on contrast texture images, where a uniformly colored pattern surrounded by a multicolor texture can be distinguished.

In the future, it would be interesting to develop a multi-gamut mapping algorithm capable of computing automatically the ink surface coverages on the recto and verso sides in order to display any pair of color images, taking into account the non-reproducible colors and trying to modify the two original images until all their colors are reproducible in the considered illumination configurations. This is a challenging task, but a tool like this seems necessary if we want that graphical designer exploit the potential of multiview recto-verso images in graphical applications.

\section{References}

[1] T. Baar, M. Shahpaski, M. V. Ortiz Segovia, "Image ghosting reduction in lenticular relief prints," Proc. SPIE 9018, paper 90180N (2014).

[2] R.D. Hersch, F. Collaud, P. Emmel, "Reproducing color images with embedded metallic patterns," Proc. SIGGRAPH'03, ACM Trans. Graph. 22, 427-436 (2003).

[3] V. Babaei, R.D. Hersch, "Color Reproduction ofMetallic-Ink Images," J. Im. Sc. Technol. 60, paper 030503 (2016).

[4] P. Pjanic, R.D. Hersch, "Color Imaging and Pattern Hiding on a Metallic Substrate," Proc. SIGGRAPH'15, ACM Trans. Graph. 34, article $130(2015)$.

[5] P. Pjanic, R.D. Hersch, "Color changing effects with anisotropic halftone prints on metal," Proc. SIGGRAPH'15, ACM Trans. Graph. 34, Article 167 (2015).

[6] R. Rossier, R. D. Hersch, "Hiding patterns with daylight fluorescent inks," Proc. IS\&T 19th Color and Imaging Conference, 223-228 (2011).

[7] S. Mazauric, M. Hébert, L. Simonot, T. Fournel, "2-flux transfer matrix model for predicting the reflectance and transmittance of duplex halftone prints," J. Opt. Soc. Am. A 31, 2775-2788 (2014).

[8] S. Mazauric, M. Hébert, T. Fournel, "Model-based Design of RectoVerso Prints Displaying Different Images According to the Illuminated Face," Color Imaging Conference (Darmstadt, Germany, 19-23 October 2015)

[9] M. Hébert, S. Mazauric, L. Simonot, "Assessing the capacity of twoflux models to predict the spectral properties of layered materials," IS\&T Electronic Imaging Symposium, Measuring, modeling, reproduction materials appearance (San Francisco, USA, 14-18 February 2015).

[10] R.D. Hersch, M. Hébert, "Base models for color halftone reproduction" in Handbook of Digital Imaging, Ed. Mickael Kriss, Wiley, New York, 1079-1132 (2015).

[11] R. D. Hersch and F. Crété, "Improving the Yule-Nielsen modified spectral Neugebauer model by dot surface coverages depending on the ink superposition conditions," Proc. SPIE 5667, 434-445 (2005).

[12] M. Hébert, R.D. Hersch, "Review of spectral reflectance prediction models for halftone prints: calibration, prediction and performance," Color Res. Appl., paper 21907 (2014).

[13] M. Hébert, P. Emmel, "Two-flux and multiflux matrix models for colored surfaces" in Handbook of Digital Imaging, Ed. Mickael Kriss, Wiley, New York, 1233-1277 (2015).

[14] M. Hébert, R.D. Hersch, "Extending the Clapper-Yule model to rough printing supports," J. Opt. Soc. Am. A 22, 1952-1967 (2005).

[15] G. Sharma, Digital Color Imaging Handbook (CRC Press, 2003), pp $30-36$.

[16] J. A. S. Viggiano, "The color of halftone tints," Proc. TAGA 37, $647-$ $661(1985)$ 
[17] M. Hébert, R.D. Hersch, "Yule-Nielsen based recto-verso color halftone transmittance prediction model," Appl. Opt. 50, 519-525 (2011).

[18] H. Kipphan, Handbook of Print Media (Springer, 2001).

[19] M. D. Fairchild, Color Appearance Models, 2nd Ed., Wiley-IS\&T, Chichester, UK (2005), p. 27.

[20] J. Machizaud, T. Fournel, "Two-out-of-two color matching based visual cryptography schemes," Optics Express 20, 22847-22859 (2012).

[21] M. Naor and A. Shamir, "Visual cryptography," Lecture Notes in Computer Science 950, 1-12 (1995).

\section{Acknowledgement}

This work was supported by the French National Research Agency (ANR) within the program "Investissements d'Avenir" (ANR-11-IDEX-0007), in the framework of the LABEX MANUTECH-SISE (ANR-10-LABX-0075) of Université de Lyon. The authors would also like to thank Dorian Saint-Pierre, Louis Duveau and Baptiste Langlois for their participation to the production of samples.

\section{Authors Biography}

Nicolas Dalloz is an MSc student at Institut d'Optique Graduate School and at Optics-Image-Vision-Multimedia master of University Jean Monnet, Saint-Etienne. He is also intern at Laboratoire Hubert Curien since May 2016. He is interested in optical methods for assessing material appearance.

Serge Mazauric completed his PhD in 2016 in the field of computational printing at the Laboratoire Hubert Curien of CNRS and University Jean Monnet of Saint-Etienne. His work focuses on optical models for applications in color reproduction.

Thierry Fournel is Professor at St Etienne University in France where he received his $P h D$ in image processing in1991. After a first decade devoted to particle image tracking techniques, his main research topics are today computational printing and visual cryptography in addition to teaching applied information theory.

Mathieu Hébert completed his PhD studies in 2006 at the Ecole Polytechnique Fédérale de Lausanne (EPFL, Switzerland) and is now assitant professor at the Institut d'Optique-Graduate School and the University of Saint-Etienne, France. His research activity is focused on optical models for predicting the appearance of colored surfaces. 